\title{
Jean Bouchet, Euvres complètes: vol. I. Le Jugement poetic de l'honneur femenin
}

\section{Michele Mastroianni}

\section{Q OpenEdition \\ 1 Journals}

\section{Edizione digitale}

URL: http://journals.openedition.org/studifrancesi/27362

DOI: 10.4000/studifrancesi.27362

ISSN: 2421-5856

\section{Editore}

Rosenberg \& Sellier

\section{Edizione cartacea}

Data di pubblicazione: 31 décembre 2006

Paginazione: $587-588$

ISSN: 0039-2944

\section{Notizia bibliografica digitale}

Michele Mastroianni, « Jean Bouchet, E Euvres complètes: vol. I. Le Jugement poetic de l'honneur femenin ", Studi Francesi [Online], 150 (L | III) | 2006, online dal 30 novembre 2015, consultato il 08 novembre 2020. URL : http://journals.openedition.org/studifrancesi/27362 ; DOI : https://doi.org/10.4000/ studifrancesi.27362

\section{Questo documento è stato generato automaticamente il 8 novembre 2020}

\section{(c) $(1) \ominus$}

Studi Francesi è distribuita con Licenza Creative Commons Attribuzione - Non commerciale - Non opere derivate 4.0 Internazionale. 


\title{
Jean Bouchet, CEuvres complètes: vol. I. Le Jugement poetic de l'honneur femenin
}

\author{
Michele Mastroianni
}

\section{NOTIZIA}

JEAN BOUCHET, CEuvres complètes: vol. I. Le Jugement poetic de l'honneur femenin, édition critique par Adrian Armstrong, Paris, Champion («Textes de la Renaissance», 101), 2006, pp. 596.

1 Il lungo poema didascalico (quasi 4000 decasillabi) di Jean Bouchet, mai più edito dopo la stampa del 1538 (Poitiers, Jean et Enguilbert de Marnef), rientra nell'ampio dibattito quattro-cinquecentesco sulla questione femminile. Opera di circostanza - ne è pretesto la commemorazione della madre di François $\mathrm{I}^{\mathrm{er}}$, Louise de Savoie - è in realtà una difesa delle donne, di cui rivendica il diritto all'educazione. L'importanza dell'educazione per le donne era già stato argomento affrontato da Bouchet nell'Histoire et cronique de Clotaire (1518) e nel Panegyric $d u$ Chevallier sans reproche (1527), a partire dall'illustrazione di exempla di valore femminile. Il Jugement, dopo aver cantato le virtù della defunta Louise, gli exploits della sua reggenza dal 1522 al 1526 e la sua attività diplomatica culminata nella pace di Cambrai, celebra le imprese e le doti di numerose donne famose al punto da essere una specie di De mulieribus claris in versi. L'opera di Bouchet è un tipico esempio della poetica dei rhétoriqueurs, di cui vengono messe in atto tutte le strategie formali e parenetiche. A. Armstrong, in un'introduzione (pp. 11-165) che è una vera e propria monografia, affronta una questione da lui definita incontournable - nel quadro della querelle des femmes cui il Jugement apporta uno dei contributi più interessanti -, la questione cioè di sapere se i testi della querelle siano anzitutto esercizi di retorica. La discussione su questo punto è stata accesa: una lettura puramente 'retorica' dei testi della querelle è stata difesa sulla base della presenza schiacciante di luoghi comuni negli argomenti dei due schieramenti, ed è stata difesa 
anche da parte di ricercatori aperti agli studi di gender, come Floyd Gray. Armstrong, in parte sulla scia di un importante lavoro di Alcuin Blamires (The Case for Women in Medieval Culture, Oxford, Clarendon Press, 1997), ritiene che «non si possa presumere che il virtuosismo formale e retorico - elemento incontestabilmente evidente in certi testi della querelle - escluda un contenuto significativo. Il fatto che si ragioni così circa $\mathrm{i}$ testi misogini o proféminins, e non su altri testi dalla forte dimensione retorica come quelli di propaganda religiosa o politica, implica francamente il rifiuto di prendere sul serio qualsiasi riflessione sulla posizione delle donne. Il che sarebbe un atteggiamento incompatibile con quanto ci hanno dimostrato importanti studi sulla relazione tra retorica e ideologia nel periodo in questione: la sofisticazione formale di un testo non ne mina necessariamente il contenuto» (pp. 33-34). Armstrong non usa il termine 'femminista', in quanto ingannevole, data la diversità delle condizioni sociali e discorsive del XVI secolo rispetto a quelle che forniscono il quadro ai diversi femminismi moderni. Sempre accogliendo una proposta di Blamires, egli preferisce usare proféminin, termine che può adattarsi molto bene alla situazione in cui un discorso teso a dimostrare - in forma di elogio - che le donne sono capaci di exploits degni di un uomo, lascia intatto un sistema di valori patriarcale, secondo il quale le qualità migliori sono identificate al maschile. Termine anche che meglio si adatta a un'epoca di principi sociali e politici oscillanti e incerti, caratterizzata da quelle tensioni e contraddizioni che ritroviamo nel Jugement di Bouchet.

2 Siamo in presenza di un'edizione critica esemplare, per rigore filologico e ricchezza di erudizione. Tuttavia, proprio per l'ampio excursus sul discorso proféminin della fine del Medioevo e del Rinascimento, questa edizione costituisce anche un contributo importante agli storici delle donne e delle mentalità, non solo nell'introduzione, ma anche nelle densissime note e, soprattutto, nell'Appendice (pp. 419-517), in cui i 126 epigrammi di elogio delle mulieres clarae, vengono minuziosamente analizzati per evidenziare gli interventi ideologici operati da Bouchet sulle fonti (fonti cui, peraltro, viene dedicata una particolare attenzione nella repertoriazione). Detto questo si deve sottolineare come l'edizione sia anche uno straordinario strumento di lavoro per lo storico della lingua, per il glossario e gli indici, ma soprattutto per la sezione (pp. 82-131) consacrata sia alle strutture linguistiche che retoriche, con minuziosa attenzione agli aspetti sintattici dello stile, alla versificazione, ai fatti morfologici, all'ortografia e alla punteggiatura. Lavoro esemplare. 